Kragujevac Journal of Mathematics

Volume 40(2) (2016), Pages 238-259.

\title{
THE HIGHT ORDER LANE-EMDEN FRACTIONAL DIFFERENTIAL SYSTEM: EXISTENCE, UNIQUENESS AND ULAM TYPE STABILITIES
}

\author{
AMELE TAIEB $^{2}$ AND ZOUBIR DAHMANI ${ }^{1}$
}

\begin{abstract}
In this paper, by considering a more general Lane-Emden system of high order fractional differential equations with two arbitrary orders in each equation, we obtain some results on the existence and uniqueness of solutions using some fixed point theorems. Furthermore, we define and study some types of Ulam stability. Some examples are presented to illustrate the main results.
\end{abstract}

\section{Introduction AND Preliminaries}

In recent years, fractional calculus has attracted great attention. It provides an excellent tool for the description of hereditary properties of various materials and processes. Moreover, the fractional differential equations theory arises in many engineering and scientific disciplines such as mechanics, physics, chemistry, biology, economics, control theory and signal processing, (see $[22,23,25]$ ). Many authors investigated the existence and uniqueness of solutions for nonlinear fractional differential equations. We refer the reader to $[1-5,10-17,21,26,33]$ for more information and applications.

On the other hand, the Ulam type stabilities for fractional differential problems are quite significant in realistic problems, numerical analysis, biology and economics. Some results concerning these fractional stabilities have been obtained in [8,19,20,34].

Let us now introduce some other important research papers related to the LaneEmden model which has inspired our work: we know that modeling of several physical phenomena, such as pattern formation, population evolution and chemical reactions

Key words and phrases. Caputo derivative, fixed point, differential equation, existence, uniqueness, Ulam-Hyers stability, generalized Ulam-Hyers stability.

2010 Mathematics Subject Classification. Primary: 30C45. Secondary: 39B72, 39B82.

Received: May 23, 2015.

Accepted: December 26, 2015. 
gives rise to the Lane-Emden differential problem [9]. Ever after, the Lane-Emden systems and other related systems have exhausted the attention of many authors $[6,7,18,27,29,30,35,36]$.

Lane-Emden model has the following form:

$$
x^{\prime \prime}(t)+\frac{a}{t} x^{\prime}(t)+f(t, x(t))=g(t), \quad t \in[0,1]
$$

with the initial conditions:

$$
x(0)=A, \quad x^{\prime}(0)=B,
$$

where $A$ and $B$ are constants, $f$ is a continuous real valued function and $g \in C([0,1])$, (see the paper of J. Serrin and H. Zou [32]).

Recently, S. M. Mechee and N. Senu proposed a numerical study of the Lane-Emden differential problem of fractional order. The imposed Lane-Emden model has a more importance in applied mathematics, mathematical physics and astrophysics. The order appeared in two different fractional order as follows [28]:

$$
D^{\alpha} y(t)+\frac{k}{t^{\alpha-\beta}} D^{\beta} y(t)+f(t, y(t))=g(t)
$$

where $t \in[0,1], k \geq 0,1<\alpha \leq 2$, and $0<\beta \leq 1$, with the initial conditions $y(0)=A, y^{\prime}(0)=B$, where $A$ and $B$ are constants, $f$ is a continuous real valued function and $g \in C([0,1])$.

Very recently, R. W. Ibrahim [19] imposed the Ulam-Hyers stability for the following singular variable Lane-Emden equation:

$$
D^{\beta}\left(D^{\alpha}+\frac{a}{t}\right) u(t)+f(t, u(t))=g(t)
$$

where $u(0)=\mu, u(1)=\nu, 0<\alpha, \beta \leq 1,0 \leq t \leq 1, a \geq 0$, and where $D^{\gamma}$ denotes the Caputo fractional derivative for $\gamma>0, f$ is continuous real valued function and $g \in C([0,1])$.

In this paper, we consider a more general and high dimensional Lane-Emden coupled system of fractional differential equations. Then, we discuss the existence, uniqueness and some types of Ulam stabilities for the proposed coupled nonlinear fractional 
system. So, let us consider:

$$
\left\{\begin{array}{c}
D^{\beta_{1}}\left(D^{\alpha_{1}}+\frac{a_{1}}{t}\right) x_{1}(t)+f_{1}\left(t, x_{1}(t), x_{2}(t), \ldots, x_{n}(t)\right)=g_{1}(t), \quad t \in J, \\
D^{\beta_{2}}\left(D^{\alpha_{2}}+\frac{a_{2}}{t}\right) x_{2}(t)+f_{2}\left(t, x_{1}(t), x_{2}(t), \ldots, x_{n}(t)\right)=g_{2}(t), \quad t \in J \\
\vdots \\
D^{\beta_{n}}\left(D^{\alpha_{n}}+\frac{a_{n}}{t}\right) x_{n}(t)+f_{n}\left(t, x_{1}(t), x_{2}(t), \ldots, x_{n}(t)\right)=g_{n}(t), \quad t \in J \\
\sum_{k=1}^{n}\left|x_{k}(0)\right|=\sum_{k=1}^{n}\left|x_{k}^{\prime}(0)\right|=\cdots=\sum_{k=1}^{n}\left|x_{k}^{(l-1)}(0)\right|=0, \\
\sum_{k=1}^{n}\left|D^{\alpha_{k}} x_{k}(0)\right|=\sum_{k=1}^{n}\left|D^{\alpha_{k+1}} x_{k}(0)\right|=\cdots=\sum_{k=1}^{n}\left|D^{\alpha_{k+l-2}} x_{k}(0)\right|=0, \\
D^{\alpha_{k+l-1}} x_{k}(1)=0, \quad k=1,2, \ldots, n,
\end{array}\right.
$$

where $l-1<\beta_{k}<l, l-1<\alpha_{k}<l, a_{k} \geq 0, l \in \mathbb{N}^{*}-\{1\}, k=1,2, \ldots, n, n \in \mathbb{N}^{*}$ and $J:=[0,1]$. The derivatives $D^{\beta_{k}}$ and $D^{\alpha_{k}}, k=1,2, \ldots, n$, are in the sense of Caputo. For each $k=1,2, \ldots, n$, the functions $f_{k}: J \times \mathbb{R}^{n} \rightarrow \mathbb{R}$ and $g_{k}: J \rightarrow \mathbb{R}$ will be specified later.

To the best of our knowledge, there are no papers that have developed the LaneEmden system in multi-variables, with arbitrary orders in each equation.

Definition 1.1 ([22,31]). The Riemann-Liouville fractional integral operator of order $\alpha>0$, for a continuous function $f$ on $[0, \infty[$ is defined as:

$$
J^{\alpha} f(t)= \begin{cases}\frac{1}{\Gamma(\alpha)} \int_{0}^{t}(t-s)^{\alpha-1} f(s) d s, & \alpha>0, \\ f(t), & \alpha=0,\end{cases}
$$

where $t \geq 0$, and $\Gamma(\alpha):=\int_{0}^{\infty} e^{-x} x^{\alpha-1} d x$.

Definition $1.2([22,31])$. The Caputo derivative of order $\alpha$ for a function $x:[0, \infty) \rightarrow$ $\mathbb{R}$, which is at least l-times differentiable can be defined as:

$$
D^{\alpha} x(t)=\frac{1}{\Gamma(l-\alpha)} \int_{0}^{t}(t-s)^{l-\alpha-1} x^{(l)}(s) d s=J^{l-\alpha} x^{(l)}(t),
$$

for $l-1<\alpha<l, l \in \mathbb{N}^{*}$.

Lemma $1.1([22])$. For $\alpha>0$, the general solution of the fractional differential equation $D^{\alpha} x(t)=0$, is given by

$$
x(t)=\sum_{j=0}^{l-1} c_{j} t^{j}
$$

where $c_{j} \in \mathbb{R}, j=0, \ldots, l-1, l=[\alpha]+1$. 
Lemma $1.2([22])$. Let $\alpha>0$. Then

$$
J^{\alpha} D^{\alpha} x(t)=x(t)+\sum_{j=0}^{l-1} c_{j} t^{j},
$$

where $c_{j} \in \mathbb{R}, j=0,1, \ldots, l-1, l=[\alpha]+1$.

Lemma $1.3([22])$. Let $q>p>0, g \in L^{1}([a, b])$. Then $D^{p} J^{q} g(t)=J^{q-p} g(t)$, $t \in[a, b]$.

Lemma 1.4 ([24], Krasnoselskii). Let $M$ be a closed convex and nonempty subset of a Banach space $X$. Let $A$ and $B$ be the operators such that

(i) $A x+B y \in M$, whenever $x, y \in M$,

(ii) $A$ is a compact and continuous,

(iii) $B$ is a contraction mapping.

Then there exists $z \in M$ such that $z=A z+B z$.

The following auxiliary result is important to give the integral solution of (1.1).

Lemma 1.5. Suppose that $\left(G_{k}\right)_{k=1}^{n} \in C(J, \mathbb{R}), J=[0,1]$ and consider the nonlinear system

$$
\left\{\begin{array}{cl}
D^{\beta_{1}}\left(D^{\alpha_{1}}+\frac{a_{1}}{t}\right) x_{1}(t)=G_{1}(t), & t \in J, \\
D^{\beta_{2}}\left(D^{\alpha_{2}}+\frac{a_{2}}{t}\right) x_{2}(t)=G_{2}(t), & t \in J, \\
\vdots & \\
D^{\beta_{n}}\left(D^{\alpha_{n}}+\frac{a_{n}}{t}\right) x_{n}(t)=G_{n}(t), & t \in J,
\end{array}\right.
$$

where $l-1<\beta_{k}, \alpha_{k}<l, a_{k} \geq 0, k=1,2, \ldots, n, l \in \mathbb{N}^{*}-\{1\}$, with the conditions:

$$
\begin{aligned}
& \sum_{k=1}^{n}\left|x_{k}(0)\right|=\sum_{k=1}^{n}\left|x_{k}^{\prime}(0)\right|=\cdots=\sum_{k=1}^{n}\left|x_{k}^{(l-1)}(0)\right|=0, \\
& \sum_{k=1}^{n}\left|D^{\alpha_{k}} x_{k}(0)\right|=\sum_{k=1}^{n}\left|D^{\alpha_{k+1}} x_{k}(0)\right|=\cdots=\sum_{k=1}^{n}\left|D^{\alpha_{k+l-2}} x_{k}(0)\right|=0,
\end{aligned}
$$

where $D^{\alpha_{k+l-1}} x_{k}(1)=0, k=1,2, \ldots, n, l \in \mathbb{N}^{*}-\{1\}$. Then, (1.2)-(1.3) has a unique solution given by $\left(x_{1}, x_{2}, \ldots, x_{n}\right)(t)$, where

$$
\begin{aligned}
x_{k}(t)= & \int_{0}^{t} \frac{(t-\tau)^{\alpha_{k}-1}}{\Gamma\left(\alpha_{k}\right)}\left(\int_{0}^{\tau} \frac{(\tau-s)^{\beta_{k}-1}}{\Gamma\left(\beta_{k}\right)} G_{k}(s) d s-\frac{a_{k}}{\tau} x_{k}(\tau)\right) d \tau \\
& -\frac{t^{\alpha_{k}+l-1}}{\Gamma\left(\alpha_{k}+l\right)} \int_{0}^{1} \frac{(1-\tau)^{\alpha_{k}-1}}{\Gamma\left(\alpha_{k}\right)}\left(\int_{0}^{\tau} \frac{(\tau-s)^{\beta_{k}-1}}{\Gamma\left(\beta_{k}\right)} G_{k}(s) d s-\frac{a_{k}}{\tau} x_{k}(\tau)\right) d \tau,
\end{aligned}
$$

and $k=1,2, \ldots, n$. 
Proof. By Applying Lemma 1.2 to the problem (1.2), we get:

$$
\left(D^{\alpha_{k}}+\frac{a_{k}}{\tau}\right) x_{k}(\tau)=\int_{0}^{\tau} \frac{(\tau-s)^{\beta_{k}-1}}{\Gamma\left(\beta_{k}\right)} G_{k}(s) d s-c_{0}^{k}-c_{1}^{k} \tau-\cdots-c_{l-1}^{k} \tau^{l-1},
$$

where $k=1,2, \ldots, n,\left(c_{j}^{k}\right)_{j=0, \ldots, l-1} \in \mathbb{R}$, and $l-1<\beta_{k}<l, l \in \mathbb{N}^{*}-\{1\}$.

Now, applying Lemma 1.2 to the last assertion, yields

$$
\begin{aligned}
x_{k}(t)= & \int_{0}^{t} \frac{(t-\tau)^{\alpha_{k}-1}}{\Gamma\left(\alpha_{k}\right)}\left(\int_{0}^{\tau} \frac{(\tau-s)^{\beta_{k}-1}}{\Gamma\left(\beta_{k}\right)} G_{k}(s) d s-\frac{a_{k}}{\tau} x_{k}(\tau)\right) d \tau \\
& -\frac{c_{0}^{k} t^{\alpha_{k}}}{\Gamma\left(\alpha_{k}+1\right)}-\frac{c_{1}^{k} t^{\alpha_{k}+1}}{\Gamma\left(\alpha_{k}+2\right)}-\cdots-\frac{(l-1) ! c_{l-1}^{k} t^{\alpha_{k}+l-1}}{\Gamma\left(\alpha_{k}+l\right)} \\
& -c_{0}^{\prime k}-c_{1}^{\prime k} t-\cdots-c_{l-1}^{k} t^{l-1}
\end{aligned}
$$

where $k=1,2, \ldots, n$ and $\left(c_{j}^{\prime k}\right)_{j=0, \ldots, l-1} \in \mathbb{R}, l-1<\alpha_{k}<l, l \in \mathbb{N}^{*}-\{1\}$.

Using Lemma 1.3 and applying the conditions (1.3), we obtain the values of $c_{j}^{k}$ and $c_{j}^{\prime k}$. Substituting the last condition in (1.5), we obtain (1.4). The proof of Lemma 1.5 is thus completed.

Now, let us introduce the Banach space:

$$
S:=\left\{\left(x_{1}, x_{2}, \ldots, x_{n}\right): x_{k} \in C(J, \mathbb{R}), \quad k=1,2, \ldots, n\right\},
$$

endowed with the norm:

$$
\begin{aligned}
\left\|\left(x_{1}, x_{2}, \ldots, x_{n}\right)\right\|_{S} & =\max \left(\left\|x_{1}\right\|,\left\|x_{2}\right\|, \ldots,\left\|x_{n}\right\|\right), \\
\left\|x_{k}\right\| & =\sup _{t \in J}\left|x_{k}(t)\right|,
\end{aligned}
$$

where $k=1,2, \ldots, n$.

\section{Main Results}

In this section, we will formulate and establish sufficient conditions for the existence and uniqueness of solutions to (1.1). Then, we continue our study by imposing some types of Ulam stability: Ulam-Hyers stability, generalized Ulam-Hyers stability and Ulam-Heyers-Rassias stability for the problem (1.1).

We begin by list the following hypotheses:

$\left(H_{1}\right)$ : There exist nonnegative constants $\left(\mu_{k}\right)_{j}, j, k=1,2, \ldots, n$, such that for all $t \in[0,1]$ and all $\left(x_{1}, x_{2}, \ldots, x_{n}\right),\left(y_{1}, y_{2}, \ldots, y_{n}\right) \in S$, we have

$$
\left|f_{k}\left(t, x_{1}, x_{2}, \ldots, x_{n}\right)-f_{k}\left(t, y_{1}, y_{2}, \ldots, y_{n}\right)\right| \leq \sum_{j=1}^{n}\left(\mu_{k}\right)_{j}\left|x_{j}-y_{j}\right| .
$$

$\left(H_{2}\right)$ : The functions $f_{k}:[0,1] \times \mathbb{R}^{n} \rightarrow \mathbb{R}$ and $g_{k}:[0,1] \rightarrow \mathbb{R}$ are continuous for each $k=1,2, \ldots, n, n \in \mathbb{N}^{*}$. 
$\left(H_{3}\right)$ : For all $k=1,2, \ldots, n, n \in \mathbb{N}^{*}$, the function $f_{k}$ maps bounded subsets of $J \times \mathbb{R}^{n}$ into relatively compact subsets of $\mathbb{R}$.

$\left(H_{4}\right)$ : There exist nonnegative constants $\left(L_{k}\right)_{k=1,2 \ldots, n}$, such that, for each $t \in J$ and all $\left(x_{1}, x_{2}, \ldots, x_{n}\right) \in \mathbb{R}^{n}$,

$$
\left|f_{k}\left(t, x_{1}, x_{2}, \ldots, x_{n}\right)\right| \leq L_{k}, \quad k=1,2, \ldots, n .
$$

$\left(H_{5}\right)$ : There exist nonnegative constants $\left(M_{k}\right)_{k=1,2 \ldots, n,}$, such that, for each $t \in J$, $\left|g_{k}(t)\right| \leq M_{k}, k=1,2, \ldots, n$.

Then, we set the following quantities:

$$
\digamma_{k}=\frac{\Gamma\left(\alpha_{k}+l\right)+1}{\Gamma\left(\alpha_{k}+l\right) \Gamma\left(\alpha_{k}+\beta_{k}+1\right)}, \quad \Lambda_{k}=\frac{\Gamma\left(\alpha_{k}+l\right)+1}{\left(\alpha_{k}+l-1\right) \Gamma\left(2 \alpha_{k}+l-1\right)},
$$

and

$$
\Sigma_{k}=\sum_{j=1}^{n}\left(\mu_{k}\right)_{j}, k=1,2, \ldots, n .
$$

2.1. Existence and Uniqueness of Solutions. The first result is based on Banach contraction principle. We have:

Theorem 2.1. Assume that hypotheses $\left(H_{1}\right),\left(H_{4}\right)$ and $\left(H_{5}\right)$ are satisfied. Then, the system (1.1) has a unique solution on $J$ provided that

$$
\lambda_{k}=: \Sigma_{k} \digamma_{k}+a_{k} \Lambda_{k}<1, \quad k=1,2, \ldots, n .
$$

Proof. Define the nonlinear operator $T: S \rightarrow S$ by

$$
\begin{aligned}
T\left(x_{1}, x_{2}, \ldots, x_{n}\right)(t):= & \left(T_{1}\left(x_{1}, x_{2}, \ldots, x_{n}\right)(t),\right. \\
& \left.T_{2}\left(x_{1}, x_{2}, \ldots, x_{n}\right)(t), \ldots, T_{n}\left(x_{1}, x_{2}, \ldots, x_{n}\right)(t)\right), \quad t \in J,
\end{aligned}
$$

where, for all $k=1,2, \ldots, n$,

$$
\begin{aligned}
T_{k}\left(x_{1}, \ldots, x_{n}\right)(t)= & \int_{0}^{t} \frac{(t-\tau)^{\alpha_{k}-1}}{\Gamma\left(\alpha_{k}\right)}\left(\int_{0}^{\tau} \frac{(\tau-s)^{\beta_{k}-1}}{\Gamma\left(\beta_{k}\right)}\right. \\
& \left.\times\left(g_{k}(s)-f_{k}\left(s, x_{1}(s), \ldots, x_{n}(s)\right)\right) d s-\frac{a_{k}}{\tau} x_{k}(\tau)\right) d \tau \\
& -\frac{t^{\alpha_{k}+l-1}}{\Gamma\left(\alpha_{k}+l\right)} \int_{0}^{1} \frac{(1-\tau)^{\alpha_{k}-1}}{\Gamma\left(\alpha_{k}\right)}\left(\int_{0}^{\tau} \frac{(\tau-s)^{\beta_{k}-1}}{\Gamma\left(\beta_{k}\right)}\right. \\
& \left.\times\left(g_{k}(s)-f_{k}\left(s, x_{1}(s), \ldots, x_{n}(s)\right)\right) d s-\frac{a_{k}}{\tau} x_{k}(\tau)\right) d \tau .
\end{aligned}
$$

For

$$
r \geq \frac{\left(M_{k}+L_{k}\right) \digamma_{k}}{1-\sigma}, \quad \lambda_{k} \leq \sigma<1
$$


we consider the set $B_{r}:=\left\{\left(x_{1}, x_{2}, \ldots, x_{n}\right) \in S ;\left\|\left(x_{1}, x_{2}, \ldots, x_{n}\right)\right\|_{S} \leq r\right\}$ and we will show that $T B_{r} \subset B_{r}$.

For $\left(x_{1}, x_{2}, \ldots, x_{n}\right) \in B_{r}$ and each $k=1,2, \ldots, n$, we have

$$
\begin{aligned}
& \left\|T_{k}\left(x_{1}, x_{2}, \ldots, x_{n}\right)\right\| \\
& \leq \sup _{t \in J} \int_{0}^{t} \frac{(t-\tau)^{\alpha_{k}-1}}{\Gamma\left(\alpha_{k}\right)}\left(\int_{0}^{\tau} \frac{(\tau-s)^{\beta_{k}-1}}{\Gamma\left(\beta_{k}\right)}\right. \\
& \quad \times\left|g_{k}(s)-f_{k}\left(s, x_{1}(s), \ldots, x_{n}(s)\right)-f_{k}(s, 0, \ldots, 0)+f_{k}(s, 0, \ldots, 0)\right| d s \\
& \left.\quad+\left|\frac{a_{k}}{\tau} x_{k}(\tau)\right|\right) d \tau \\
& +\sup _{t \in J} \frac{t^{\alpha_{k}+l-1} \Gamma\left(\alpha_{k}+l\right)}{\int_{0}^{1}} \frac{(1-\tau)^{\alpha_{k}-1}}{\Gamma\left(\alpha_{k}\right)}\left(\int_{0}^{\tau} \frac{(\tau-s)^{\beta_{k}-1}}{\Gamma\left(\beta_{k}\right)}\right. \\
& \quad \times\left|g_{k}(s)-f_{k}\left(s, x_{1}(s), \ldots, x_{n}(s)\right)-f_{k}(s, 0, \ldots, 0)+f_{k}(s, 0, \ldots, 0)\right| d s \\
& \left.\quad+\left|\frac{a_{k}}{\tau} x_{k}(\tau)\right|\right) d \tau .
\end{aligned}
$$

The hypotheses $\left(H_{4}\right)$ and $\left(H_{5}\right)$ allow us to write

$$
\begin{aligned}
& \left\|T_{k}\left(x_{1}, x_{2}, \ldots, x_{n}\right)\right\| \\
\leq \sup _{t \in J} & \int_{0}^{t} \frac{(t-\tau)^{\alpha_{k}-1}}{\Gamma\left(\alpha_{k}\right)}\left(\int_{0}^{\tau} \frac{(\tau-s)^{\beta_{k}-1}}{\Gamma\left(\beta_{k}\right)} d s\right) d \tau\left(M_{k}+L_{k}+\Sigma_{k}\left\|\left(x_{1}, x_{2}, \ldots, x_{n}\right)\right\|_{S}\right) \\
+ & \sup _{t \in J} \int_{0}^{t} \frac{(t-\tau)^{\alpha_{k}-1}}{\Gamma\left(\alpha_{k}\right)} \frac{1}{\tau} d \tau a_{k}\left\|\left(x_{1}, x_{2}, \ldots, x_{n}\right)\right\|_{S} \\
+ & \sup _{t \in J} \frac{t^{\alpha_{k}+l-1}}{\Gamma\left(\alpha_{k}+l\right)} \int_{0}^{1} \frac{(1-\tau)^{\alpha_{k}-1}}{\Gamma\left(\alpha_{k}\right)}\left(\int_{0}^{\tau} \frac{(\tau-s)^{\beta_{k}-1}}{\Gamma\left(\beta_{k}\right)} d s\right) d \tau \\
& \times\left(M_{k}+L_{k}+\Sigma_{k}\left\|\left(x_{1}, x_{2}, \ldots, x_{n}\right)\right\|_{S}\right) \\
+ & \sup _{t \in J} \frac{t^{\alpha_{k}+l-1}}{\Gamma\left(\alpha_{k}+l\right)} \int_{0}^{1} \frac{(1-\tau)^{\alpha_{k}-1}}{\Gamma\left(\alpha_{k}\right)} \frac{1}{\tau} d \tau a_{k}\left\|\left(x_{1}, x_{2}, \ldots, x_{n}\right)\right\|_{S} .
\end{aligned}
$$

Therefore, by $\left(H_{1}\right)$ 


$$
\begin{aligned}
& \left\|T_{k}\left(x_{1}, x_{2}, \ldots, x_{n}\right)\right\| \\
& \leq\left(M_{k}+L_{k}+\Sigma_{k}\left\|\left(x_{1}, x_{2}, \ldots, x_{n}\right)\right\|_{S}\right) \\
& \quad \times\left(1+\frac{1}{\Gamma\left(\alpha_{k}+l\right)}\right) \int_{0}^{1} \frac{(1-\tau)^{\alpha_{k}-1}}{\Gamma\left(\alpha_{k}\right)}\left(\int_{0}^{\tau} \frac{(\tau-s)^{\beta_{k}-1}}{\Gamma\left(\beta_{k}\right)} d s\right) d \tau \\
& \quad+a_{k}\left(1+\frac{1}{\Gamma\left(\alpha_{k}+l\right)}\right)\left\|\left(x_{1}, x_{2}, \ldots, x_{n}\right)\right\|_{S} \int_{0}^{1} \frac{(1-\tau)^{\alpha_{k}-1}}{\Gamma\left(\alpha_{k}\right)} \tau^{\alpha_{k}+l-2} d \tau .
\end{aligned}
$$

Hence,

$$
\begin{aligned}
& \left\|T_{k}\left(x_{1}, x_{2}, \ldots, x_{n}\right)\right\| \\
& \leq\left(M_{k}+L_{k}+\Sigma_{k} r\right)\left(\frac{1}{\Gamma\left(\alpha_{k}\right) \Gamma\left(\beta_{k}+1\right)}+\frac{1}{\Gamma\left(\alpha_{k}\right) \Gamma\left(\beta_{k}+1\right) \Gamma\left(\alpha_{k}+l\right)}\right) \\
& \quad \times \int_{0}^{1}(1-\tau)^{\alpha_{k}-1} \tau^{\beta_{k}} d \tau \\
& +a_{k} r\left(\frac{1}{\Gamma\left(\alpha_{k}\right)}+\frac{1}{\Gamma\left(\alpha_{k}\right) \Gamma\left(\alpha_{k}+l\right)}\right) \int_{0}^{1}(1-\tau)^{\alpha_{k}-1} \tau^{\alpha_{k}+l-2} d \tau .
\end{aligned}
$$

Using the Beta function, we get

$$
\begin{aligned}
& \left\|T_{k}\left(x_{1}, x_{2}, \ldots, x_{n}\right)\right\| \\
& \leq\left(M_{k}+L_{k}+\Sigma_{k} r\right)\left(\frac{1}{\Gamma\left(\alpha_{k}\right) \Gamma\left(\beta_{k}+1\right)}+\frac{1}{\Gamma\left(\alpha_{k}+l\right) \Gamma\left(\alpha_{k}\right) \Gamma\left(\beta_{k}+1\right)}\right) \\
& \quad \times \beta\left(\alpha_{k}, \beta_{k}+1\right)+a_{k} r\left(\frac{1}{\Gamma\left(\alpha_{k}\right)}+\frac{1}{\Gamma\left(\alpha_{k}\right) \Gamma\left(\alpha_{k}+l\right)}\right) \beta\left(\alpha_{k}, \alpha_{k}+l-1\right) .
\end{aligned}
$$

By (2.3), we obtain

$$
\left\|T_{k}\left(x_{1}, x_{2}, \ldots, x_{n}\right)\right\| \leq(1-\sigma) r+\lambda_{k} r=\left(1-\sigma+\lambda_{k}\right) r
$$

and

$$
\left\|T_{k}\left(x_{1}, x_{2}, \ldots, x_{n}\right)\right\| \leq r
$$

where $k=1,2, \ldots, n$.

Thus,

$$
\left\|T\left(x_{1}, x_{2}, \ldots, x_{n}\right)\right\|_{S} \leq r .
$$

We need to prove that $T$ is a contractive. 
For $\left(x_{1}, x_{2}, \ldots, x_{n}\right),\left(y_{1}, y_{2}, \ldots, y_{n}\right) \in S$ and for all $t \in J$, we have:

$$
\begin{aligned}
& \left\|T_{k}\left(x_{1}, x_{2}, \ldots, x_{n}\right)-T_{k}\left(y_{1}, y_{2}, \ldots, y_{n}\right)\right\| \\
& \leq \sup _{t \in J} \int_{0}^{t} \frac{(t-\tau)^{\alpha_{k}-1}}{\Gamma\left(\alpha_{k}\right)}\left(\int_{0}^{\tau} \frac{(\tau-s)^{\beta_{k}-1}}{\Gamma\left(\beta_{k}\right)}\right. \\
& \quad \times\left|f_{k}\left(s, x_{1}(s), \ldots, x_{n}(s)\right)-f_{k}\left(s, y_{1}(s), \ldots, y_{n}(s)\right)\right| d s \\
& \left.\quad+\frac{a_{k}}{\tau}\left|x_{k}(\tau)-y_{k}(\tau)\right|\right) d \tau \\
& +\sup _{t \in J} \frac{t^{\alpha_{k}+l-1}}{\Gamma\left(\alpha_{k}+l\right)} \int_{0}^{1} \frac{(1-\tau)^{\alpha_{k}-1}}{\Gamma\left(\alpha_{k}\right)}\left(\int_{0}^{\tau} \frac{(\tau-s)^{\beta_{k}-1}}{\Gamma\left(\beta_{k}\right)}\right. \\
& \quad \times\left|f_{k}\left(s, x_{1}(s), \ldots, x_{n}(s)\right)-f_{k}\left(s, y_{1}(s), \ldots, y_{n}(s)\right)\right| d s \\
& \left.\quad+\frac{a_{k}}{\tau}\left|x_{k}(\tau)-y_{k}(\tau)\right|\right) d \tau .
\end{aligned}
$$

Thanks to $\left(H_{1}\right)$, we can write

$$
\begin{aligned}
& \left\|T_{k}\left(x_{1}, x_{2}, \ldots, x_{n}\right)-T_{k}\left(y_{1}, y_{2}, \ldots, y_{n}\right)\right\| \\
& \leq \sup _{t \in J} \int_{0}^{t} \frac{(t-\tau)^{\alpha_{k}-1}}{\Gamma\left(\alpha_{k}\right)}\left(\int_{0}^{\tau} \frac{(\tau-s)^{\beta_{k}-1}}{\Gamma\left(\beta_{k}\right)} d s\right) d \tau \Sigma_{k}\left\|\left(x_{1}-y_{1}, x_{2}-y_{2}, \ldots, x_{n}-y_{n}\right)\right\|_{S} \\
& \quad+\sup _{t \in J} \int_{0}^{t} \frac{(t-\tau)^{\alpha_{k}-1}}{\Gamma\left(\alpha_{k}\right)} \frac{1}{\tau} d \tau a_{k}\left\|\left(x_{1}-y_{1}, x_{2}-y_{2}, \ldots, x_{n}-y_{n}\right)\right\|_{S}+\sup _{t \in J} \frac{t^{\alpha_{k}+l-1}}{\Gamma\left(\alpha_{k}+l\right)} \\
& \quad \times \int_{0}^{1} \frac{(1-\tau)^{\alpha_{k}-1}}{\Gamma\left(\alpha_{k}\right)}\left(\int_{0}^{\tau} \frac{(\tau-s)^{\beta_{k}-1}}{\Gamma\left(\beta_{k}\right)} d s\right) d \tau \Sigma_{k}\left\|\left(x_{1}-y_{1}, x_{2}-y_{2}, \ldots, x_{n}-y_{n}\right)\right\|_{S} \\
& \quad+\sup _{t \in J} \frac{t^{\alpha_{k}+l-1}}{\Gamma\left(\alpha_{k}+l\right)} \int_{0}^{1} \frac{(1-\tau)^{\alpha_{k}-1}}{\Gamma\left(\alpha_{k}\right)} \frac{1}{\tau} d \tau a_{k}\left\|\left(x_{1}-y_{1}, x_{2}-y_{2}, \ldots, x_{n}-y_{n}\right)\right\|_{S} .
\end{aligned}
$$

Consequently,

$$
\begin{aligned}
& \left\|T_{k}\left(x_{1}, x_{2}, \ldots, x_{n}\right)-T_{k}\left(y_{1}, y_{2}, \ldots, y_{n}\right)\right\| \\
\leq & \Sigma_{k} \frac{\Gamma\left(\alpha_{k}+l\right)+1}{\Gamma\left(\alpha_{k}+l\right)}\left\|\left(x_{1}-y_{1}, x_{2}-y_{2}, \ldots, x_{n}-y_{n}\right)\right\|_{S}
\end{aligned}
$$




$$
\begin{aligned}
& \quad \times \int_{0}^{1} \frac{(1-\tau)^{\alpha_{k}-1}}{\Gamma\left(\alpha_{k}\right)}\left(\int_{0}^{\tau} \frac{(\tau-s)^{\beta_{k}-1}}{\Gamma\left(\beta_{k}\right)} d s\right) d \tau \\
& +a_{k} \frac{\Gamma\left(\alpha_{k}+l\right)+1}{\Gamma\left(\alpha_{k}+l\right)}\left\|\left(x_{1}-y_{1}, x_{2}-y_{2}, \ldots, x_{n}-y_{n}\right)\right\|_{S} \\
& \quad \times \int_{0}^{1} \frac{(1-\tau)^{\alpha_{k}-1}}{\Gamma\left(\alpha_{k}\right)} \tau^{\alpha_{k}+l-2} d \tau \\
& \leq \lambda_{k}\left\|\left(x_{1}-y_{1}, x_{2}-y_{2}, \ldots, x_{n}-y_{n}\right)\right\|_{S},
\end{aligned}
$$

which implies that

$$
\begin{aligned}
& \left\|T\left(x_{1}, x_{2}, \ldots, x_{n}\right)-T\left(y_{1}, y_{2}, \ldots, y_{n}\right)\right\|_{S} \\
\leq & \max \left(\lambda_{1}, \lambda_{2}, \ldots, \lambda_{n}\right)\left\|\left(x_{1}-y_{1}, x_{2}-y_{2}, \ldots, x_{n}-y_{n}\right)\right\|_{S},
\end{aligned}
$$

where, $k=1,2, \ldots, n$.

Then by (2.2), $T$ is contractive.

Theorem 2.2. Assume that the hypotheses $\left(H_{i}\right)_{i=1,2, \ldots, 5}$ and the inequalities

$$
C_{k}:=\frac{\lambda_{k}}{\Gamma\left(\alpha_{k}+l\right)+1}<1, \quad k=1,2, \ldots, n,
$$

are satisfied. Then system (1.1) has at least one solution on J.

Proof. On $B_{R}$, such that

$$
R \geq \frac{\left(M_{k}+L_{k}\right) \digamma_{k}}{1-a_{k} \Lambda_{k}}, \quad a_{k} \Lambda_{k} \neq 1
$$

we define the operators $P$ and $Q$ as follows:

$$
\begin{aligned}
& P\left(x_{1}, x_{2}, \ldots, x_{n}\right)(t):= \\
& \quad\left(P_{1}\left(x_{1}, x_{2}, \ldots, x_{n}\right)(t), P_{2}\left(x_{1}, x_{2}, \ldots, x_{n}\right)(t), \ldots, P_{n}\left(x_{1}, x_{2}, \ldots, x_{n}\right)(t)\right), \\
& Q\left(x_{1}, x_{2}, \ldots, x_{n}\right)(t):= \\
& \quad\left(Q_{1}\left(x_{1}, x_{2}, \ldots, x_{n}\right)(t), Q_{2}\left(x_{1}, x_{2}, \ldots, x_{n}\right)(t), \ldots, Q_{n}\left(x_{1}, x_{2}, \ldots, x_{n}\right)(t)\right) .
\end{aligned}
$$

For each $k=1,2, \ldots, n$,

$$
\begin{aligned}
& P_{k}\left(x_{1}, x_{2}, \ldots, x_{n}\right)(t):= \\
& \int_{0}^{t} \frac{(t-\tau)^{\alpha_{k}-1}}{\Gamma\left(\alpha_{k}\right)}\left(\int_{0}^{\tau} \frac{(\tau-s)^{\beta_{k}-1}}{\Gamma\left(\beta_{k}\right)}\left(g_{k}(s)-f_{k}\left(s, x_{1}(s), \ldots, x_{n}(s)\right)\right) d s-\frac{a_{k}}{\tau} x_{k}(\tau)\right) d \tau,
\end{aligned}
$$

and 


$$
\begin{aligned}
Q_{k}\left(x_{1}, x_{2}, \ldots, x_{n}\right)(t):=- & \frac{t^{\alpha_{k}+l-1}}{\Gamma\left(\alpha_{k}+l\right)} \int_{0}^{1} \frac{(1-\tau)^{\alpha_{k}-1}}{\Gamma\left(\alpha_{k}\right)}\left(\int_{0}^{\tau} \frac{(\tau-s)^{\beta_{k}-1}}{\Gamma\left(\beta_{k}\right)}\right. \\
& \left.\times\left(g_{k}(s)-f_{k}\left(s, x_{1}(s), \ldots, x_{n}(s)\right)\right) d s-\frac{a_{k}}{\tau} x_{k}(\tau)\right) d \tau .
\end{aligned}
$$

For $\left(x_{1}, x_{2}, \ldots, x_{n}\right),\left(y_{1}, y_{2}, \ldots, y_{n}\right) \in B_{R}$ and for each $k=1,2, \ldots, n$, we can write

$$
\begin{aligned}
& \| P_{k}\left(x_{1}, x_{2}, \ldots, x_{n}\right)+Q_{k}\left(y_{1}, y_{2}, \ldots, y_{n}\right) \mid \\
& \leq \sup _{t \in J} \int_{0}^{t} \frac{(t-\tau)^{\alpha_{k}-1}}{\Gamma\left(\alpha_{k}\right)}\left(\int_{0}^{\tau} \frac{(\tau-s)^{\beta_{k}-1}}{\Gamma\left(\beta_{k}\right)}\right. \\
& \left.\quad \times\left(\left|g_{k}(s)\right|+\left|f_{k}\left(s, x_{1}(s), x_{2}(s), \ldots, x_{n}(s)\right)\right|\right) d s+\left|\frac{a_{k}}{\tau} x_{k}(\tau)\right|\right) d \tau \\
& +\sup _{t \in J} \frac{t^{\alpha_{k}+l-1}}{\Gamma\left(\alpha_{k}+l\right)} \int_{0}^{1} \frac{(1-\tau)^{\alpha_{k}-1}}{\Gamma\left(\alpha_{k}\right)}\left(\int_{0}^{\tau} \frac{(\tau-s)^{\beta_{k}-1}}{\Gamma\left(\beta_{k}\right)}\right. \\
& \left.\quad \times\left(\left|g_{k}(s)\right|+\left|f_{k}\left(s, y_{1}(s), y_{2}(s), \ldots, y_{n}(s)\right)\right|\right) d s+\left|\frac{a_{k}}{\tau} y_{k}(\tau)\right|\right) d \tau .
\end{aligned}
$$

Using $\left(H_{4}\right)$ and $\left(H_{5}\right)$, we obtain

$$
\begin{aligned}
& \left\|P_{k}\left(x_{1}, x_{2}, \ldots, x_{n}\right)+Q_{k}\left(y_{1}, y_{2}, \ldots, y_{n}\right)\right\| \\
& \leq \sup _{t \in J} \int_{0}^{t} \frac{(t-\tau)^{\alpha_{k}-1}}{\Gamma\left(\alpha_{k}\right)}\left(\int_{0}^{\tau} \frac{(\tau-s)^{\beta_{k}-1}}{\Gamma\left(\beta_{k}\right)} d s\right) d \tau\left(M_{k}+L_{k}\right) \\
& \quad+\sup _{t \in J} \int_{0}^{t} \frac{(t-\tau)^{\alpha_{k}-1}}{\Gamma\left(\alpha_{k}\right)} \frac{1}{\tau} d \tau a_{k}\left\|\left(x_{1}, x_{2}, \ldots, x_{n}\right)\right\|_{S} \\
& +\sup _{t \in J} \frac{t^{\alpha_{k}+l-1}}{\Gamma\left(\alpha_{k}+l\right)} \int_{0}^{1} \frac{(1-\tau)^{\alpha_{k}-1}}{\Gamma\left(\alpha_{k}\right)}\left(\int_{0}^{\tau} \frac{(\tau-s)^{\beta_{k}-1}}{\Gamma\left(\beta_{k}\right)} d s\right) d \tau\left(M_{k}+L_{k}\right) \\
& \quad+\sup _{t \in J} \frac{t^{\alpha_{k}+l-1}}{\Gamma\left(\alpha_{k}+l\right)} \int_{0}^{1} \frac{(1-\tau)^{\alpha_{k}-1}}{\Gamma\left(\alpha_{k}\right)} \frac{1}{\tau} d \tau a_{k}\left\|\left(y_{1}, y_{2}, \ldots, y_{n}\right)\right\|_{S} .
\end{aligned}
$$

And then, 


$$
\begin{aligned}
& \left\|P_{k}\left(x_{1}, x_{2}, \ldots, x_{n}\right)+Q_{k}\left(y_{1}, y_{2}, \ldots, y_{n}\right)\right\| \\
& \leq\left(M_{k}+L_{k}\right)\left(1+\frac{1}{\Gamma\left(\alpha_{k}+l\right)}\right) \int_{0}^{1} \frac{(1-\tau)^{\alpha_{k}-1}}{\Gamma\left(\alpha_{k}\right)}\left(\int_{0}^{\tau} \frac{(\tau-s)^{\beta_{k}-1}}{\Gamma\left(\beta_{k}\right)} d s\right) d \tau \\
& \quad+a_{k} R\left(1+\frac{1}{\Gamma\left(\alpha_{k}+l\right)}\right) \int_{0}^{1} \frac{(1-\tau)^{\alpha_{k}-1}}{\Gamma\left(\alpha_{k}\right)} \tau^{\alpha_{k}+l-2} d \tau \\
& \leq\left(\frac{M_{k}+L_{k}}{\Gamma\left(\alpha_{k}\right) \Gamma\left(\beta_{k}+1\right)}+\frac{M_{k}+L_{k}}{\Gamma\left(\alpha_{k}\right) \Gamma\left(\beta_{k}+1\right) \Gamma\left(\alpha_{k}+l\right)}\right) \int_{0}^{1}(1-\tau)^{\alpha_{k}-1} \tau^{\beta_{k}} d \tau \\
& \quad+a_{k} R\left(\frac{1}{\Gamma\left(\alpha_{k}\right)}+\frac{1}{\Gamma\left(\alpha_{k}\right) \Gamma\left(\alpha_{k}+l\right)}\right) \int_{0}^{1}(1-\tau)^{\alpha_{k}-1} \tau^{\alpha_{k}+l-2} d \tau .
\end{aligned}
$$

Such that $R \geq\left(M_{k}+L_{k}\right) F_{k} /\left(1-\alpha_{k} \Lambda_{k}\right), \alpha_{k} \Lambda_{k} \neq 1$, we have

$$
\begin{aligned}
\left\|P_{k}\left(x_{1}, x_{2}, \ldots, x_{n}\right)+Q_{k}\left(y_{1}, y_{2}, \ldots, y_{n}\right)\right\| \leq & \left(M_{k}+L_{k}\right) \frac{\Gamma\left(\alpha_{k}+l\right)+1}{\Gamma\left(\alpha_{k}+l\right) \Gamma\left(\alpha_{k}+\beta_{k}+1\right)} \\
& +a_{k} R \frac{\left(\Gamma\left(\alpha_{k}+l\right)+1\right)}{\left(\alpha_{k}+l-1\right) \Gamma\left(2 \alpha_{k}+l-1\right)} \\
= & \left(M_{k}+L_{k}\right) \digamma_{k}+a_{k} \Lambda_{k} R \leq R .
\end{aligned}
$$

Consequently,

$$
\left\|P\left(x_{1}, x_{2}, \ldots, x_{n}\right)+Q\left(y_{1}, y_{2}, \ldots, y_{n}\right)\right\|_{S} \leq R .
$$

Thus, $P\left(x_{1}, x_{2}, \ldots, x_{n}\right)+Q\left(y_{1}, y_{2}, \ldots, y_{n}\right) \in B_{R}$.

We proceed to prove that $Q$ is a contraction mapping in $B_{R}$. For $\left(x_{1}, x_{2}, \ldots, x_{n}\right),\left(y_{1}, y_{2}, \ldots, y_{n}\right) \in B_{R}$ and for each $k=1,2, \ldots, n$, we obtain:

$$
\begin{aligned}
& \left\|Q_{k}\left(x_{1}, x_{2}, \ldots, x_{n}\right)-Q_{k}\left(y_{1}, y_{2}, \ldots, y_{n}\right)\right\| \\
& \leq \sup _{t \in J} \frac{t^{\alpha_{k}+l-1}}{\Gamma\left(\alpha_{k}+l\right)} \int_{0}^{1} \frac{(1-\tau)^{\alpha_{k}-1}}{\Gamma\left(\alpha_{k}\right)} \int_{0}^{\tau} \frac{(\tau-s)^{\beta_{k}-1}}{\Gamma\left(\beta_{k}\right)} \\
& \quad \times\left|f_{k}\left(s, x_{1}(s), \ldots, x_{n}(s)\right)-f_{k}\left(s, y_{1}(s), \ldots, y_{n}(s)\right)\right| d s \\
& \quad+\frac{a_{k}}{\tau}\left|x_{k}(\tau)-y_{k}(\tau)\right|
\end{aligned}
$$

Using $\left(H_{1}\right)$, we get

$$
\begin{aligned}
& \left\|Q_{k}\left(x_{1}, x_{2}, \ldots, x_{n}\right)-Q_{k}\left(y_{1}, y_{2}, \ldots, y_{n}\right)\right\| \\
\leq & \frac{\Sigma_{k}}{\Gamma\left(\alpha_{k}+l\right)}\left\|\left(x_{1}-y_{1}, x_{2}-y_{2}, \ldots, x_{n}-y_{n}\right)\right\|_{S}
\end{aligned}
$$




$$
\begin{aligned}
& \times \int_{0}^{1} \frac{(1-\tau)^{\alpha_{k}-1}}{\Gamma\left(\alpha_{k}\right)}\left(\int_{0}^{\tau} \frac{(\tau-s)^{\beta_{k}-1}}{\Gamma\left(\beta_{k}\right)} d s\right) d \tau \\
& +\frac{a_{k}}{\Gamma\left(\alpha_{k}+l\right)}\left\|\left(x_{1}-y_{1}, x_{2}-y_{2}, \ldots, x_{n}-y_{n}\right)\right\|_{S} \int_{0}^{1} \frac{(1-\tau)^{\alpha_{k}-1}}{\Gamma\left(\alpha_{k}\right)} \tau^{\alpha_{k}+l-2} d \tau \\
\leq & \frac{\Sigma_{k}\left\|\left(x_{1}-y_{1}, x_{2}-y_{2}, \ldots, x_{n}-y_{n}\right)\right\|_{S}}{\Gamma\left(\alpha_{k}+l\right) \Gamma\left(\alpha_{k}\right) \Gamma\left(\beta_{k}+1\right)} \int_{0}^{1}(1-\tau)^{\alpha_{k}-1} \tau^{\beta_{k}} d \tau \\
& +\frac{a_{k}\left\|\left(x_{1}-y_{1}, x_{2}-y_{2}, \ldots, x_{n}-y_{n}\right)\right\|_{S}}{\Gamma\left(\alpha_{k}+l\right) \Gamma\left(\alpha_{k}\right)} \int_{0}^{1}(1-\tau)^{\alpha_{k}-1} \tau^{\alpha_{k}+l-2} d \tau \\
\leq & \frac{\Sigma_{k}\left\|\left(x_{1}-y_{1}, x_{2}-y_{2}, \ldots, x_{n}-y_{n}\right)\right\|_{S}}{\Gamma\left(\alpha_{k}+l\right) \Gamma\left(\alpha_{k}\right) \Gamma\left(\beta_{k}+1\right)} \beta\left(\alpha_{k}, \beta_{k}+1\right) \\
& +\frac{a_{k}\left\|\left(x_{1}-y_{1}, x_{2}-y_{2}, \ldots, x_{n}-y_{n}\right)\right\|_{S}}{\Gamma\left(\alpha_{k}+l\right) \Gamma\left(\alpha_{k}\right)} \beta\left(\alpha_{k}, \alpha_{k}+l-1\right) .
\end{aligned}
$$

Then,

$$
\begin{aligned}
& \quad\left\|Q_{k}\left(x_{1}, x_{2}, \ldots, x_{n}\right)-Q_{k}\left(y_{1}, y_{2}, \ldots, y_{n}\right)\right\| \\
& \leq \frac{\Sigma_{k}\left\|\left(x_{1}-y_{1}, x_{2}-y_{2}, \ldots, x_{n}-y_{n}\right)\right\|_{S}}{\Gamma\left(\alpha_{k}+l\right) \Gamma\left(\alpha_{k}+\beta_{k}+1\right)} \\
& \quad+\frac{a_{k} \Gamma\left(\alpha_{k}+l-1\right)\left\|\left(x_{1}-y_{1}, x_{2}-y_{2}, \ldots, x_{n}-y_{n}\right)\right\|_{S}}{\Gamma\left(\alpha_{k}+l\right) \Gamma\left(2 \alpha_{k}+l-1\right)} \\
& \leq\left(\frac{\Sigma_{k}}{\Gamma\left(\alpha_{k}+l\right) \Gamma\left(\alpha_{k}+\beta_{k}+1\right)}+\frac{a_{k}}{\left(\alpha_{k}+l-1\right) \Gamma\left(2 \alpha_{k}+l-1\right)}\right) \\
& \quad \times\left\|\left(x_{1}-y_{1}, x_{2}-y_{2}, \ldots, x_{n}-y_{n}\right)\right\|_{S} .
\end{aligned}
$$

Thus,

$$
\begin{gathered}
\left\|Q\left(x_{1}, x_{2}, \ldots, x_{n}\right)-Q\left(y_{1}, y_{2}, \ldots, y_{n}\right)\right\|_{S} \\
\leq \max _{1 \leq k \leq n} C_{k}\left\|\left(x_{1}-y_{1}, x_{2}-y_{2}, \ldots, x_{n}-y_{n}\right)\right\|_{S} .
\end{gathered}
$$

Thanks to (2.2) and (2.5), we conclude that $Q$ is a contractive.

The hypothesis $\left(H_{2}\right)$ implies that $P$ is continuous. Then, for all $\left(x_{1}, x_{2}, \ldots, x_{n}\right) \in$ $B_{R}$ and each $t \in J$, we obtain

$$
\begin{aligned}
& \left\|P_{k}\left(x_{1}, x_{2}, \ldots, x_{n}\right)\right\| \\
\leq & \sup _{t \in J} \int_{0}^{t} \frac{(t-\tau)^{\alpha_{k}-1}}{\Gamma\left(\alpha_{k}\right)}\left(\int_{0}^{\tau} \frac{(\tau-s)^{\beta_{k}-1}}{\Gamma\left(\beta_{k}\right)}\right.
\end{aligned}
$$




$$
\begin{aligned}
& \left.\quad \times\left(\left|g_{k}(s)\right|+\left|f_{k}\left(s, x_{1}(s), x_{2}(s), \ldots, x_{n}(s)\right)\right|\right) d s+\left|\frac{a_{k}}{\tau} x_{k}(\tau)\right|\right) d \tau \\
& \leq \sup _{t \in J} \int_{0}^{t} \frac{(t-\tau)^{\alpha_{k}-1}}{\Gamma\left(\alpha_{k}\right)}\left(\int_{0}^{\tau} \frac{(\tau-s)^{\beta_{k}-1}}{\Gamma\left(\beta_{k}\right)} d s\right) d \tau\left(M_{k}+L_{k}\right) \\
& \quad+\sup _{t \in J} \int_{0}^{t} \frac{(t-\tau)^{\alpha_{k}-1}}{\Gamma\left(\alpha_{k}\right)} \tau^{\alpha_{k}-l-2} d \tau a_{k}\left\|\left(x_{1}, x_{2}, \ldots, x_{n}\right)\right\|_{S} \\
& \leq \frac{M_{k}+L_{k}}{\Gamma\left(\alpha_{k}\right) \Gamma\left(\beta_{k}+1\right)} \int_{0}^{1}(1-\tau)^{\alpha_{k}-1} \tau^{\beta_{k}} d \tau+a_{k} R \frac{1}{\Gamma\left(\alpha_{k}\right)} \int_{0}^{1}(1-\tau)^{\alpha_{k}-1} \tau^{\alpha_{k}-l-2} d \tau \\
& \leq \frac{M_{k}+L_{k}}{\Gamma\left(\alpha_{k}+\beta_{k}+1\right)}+\frac{\Gamma\left(\alpha_{k}+l-1\right)}{\Gamma\left(2 \alpha_{k}+l-1\right)} a_{k} R .
\end{aligned}
$$

And by (2.6), we get

$$
\left\|P\left(x_{1}, x_{2}, \ldots, x_{n}\right)\right\|_{S} \leq R .
$$

Therefore, $P$ is uniformly bounded on $B_{R}$. Furthermore, we show that $P$ is a compact operator in $B_{R}$.

Let $0 \leq t_{1}<t_{2} \leq 1$ and $\left(x_{1}, x_{2}, \ldots, x_{n}\right) \in B_{R}$, then

$$
\begin{aligned}
& \left\|P_{k}\left(x_{1}, x_{2}, \ldots, x_{n}\right)\left(t_{2}\right)-P_{k}\left(x_{1}, x_{2}, \ldots, x_{n}\right)\left(t_{1}\right)\right\| \\
\leq & \sup _{t \in J}\left|\int_{0}^{t_{2}}\left(t_{1}-\tau\right)^{\alpha_{k}-1} \tau^{\beta_{k}} d \tau-\int_{0}^{t_{1}}\left(t_{2}-\tau\right)^{\alpha_{k}-1} \tau^{\beta_{k}} d \tau\right| \frac{M_{k}+L_{k}}{\Gamma\left(\alpha_{k}\right) \Gamma\left(\beta_{k}+1\right)} \\
& +\frac{a_{k} R}{\Gamma\left(\alpha_{k}\right)} \sup _{t \in J}\left|\int_{0}^{t_{2}}\left(t_{2}-\tau\right)^{\alpha_{k}-1} \tau^{\alpha_{k}-l-2} d \tau-\int_{0}^{t_{1}}\left(t_{1}-\tau\right)^{\alpha_{k}-1} \tau^{\alpha_{k}-l-2} d \tau\right| \\
= & \frac{M_{k}+L_{k}}{\Gamma\left(\alpha_{k}+\beta_{k}+1\right)}\left(t_{2}^{\alpha_{k}+\beta_{k}}-t_{1}^{\alpha_{k}+\beta_{k}}\right)+\frac{a_{k} R \Gamma\left(\alpha_{k}+l-1\right)}{\Gamma\left(2 \alpha_{k}+l-1\right)}\left(t_{2}^{2 \alpha_{k}+l-2}-t_{1}^{2 \alpha_{k}+l-2}\right) .
\end{aligned}
$$

Thus,

$$
\begin{aligned}
& \left\|P\left(x_{1}, x_{2}, \ldots, x_{n}\right)\left(t_{2}\right)-P\left(x_{1}, x_{2}, \ldots, x_{n}\right)\left(t_{1}\right)\right\|_{S} \\
\leq & \max _{1 \leq k \leq n} \frac{M_{k}+L_{k}}{\Gamma\left(\alpha_{k}+\beta_{k}+1\right)}\left(t_{2}^{\alpha_{k}+\beta_{k}}-t_{1}^{\alpha_{k}+\beta_{k}}\right) \\
& +\max _{1 \leq k \leq n} \frac{a_{k} R \Gamma\left(\alpha_{k}+l-1\right)}{\Gamma\left(2 \alpha_{k}+l-1\right)}\left(t_{2}^{2 \alpha_{k}+l-2}-t_{1}^{2 \alpha_{k}+l-2}\right) .
\end{aligned}
$$

The right-hand side of the above inequalities (2.7) is independent of $\left(x_{1}, x_{2}, \ldots, x_{n}\right)$ and tend to zero as $t_{2} \rightarrow t_{1}$. Therefore, $P$ is equicontinuous. Furthermore, by the hypothesis $\left(H_{3}\right), P$ is relatively compact on $B_{R}$. By Arzela-Ascoli Theorem, $P$ is a 
compact operator on $B_{R}$. Then thanks to Lmma 1.6, we can state that (1.1) has at least one solution on $J$. Theorem 2.2 is thus proved.

2.2. Ulam Stabilities. We introduce the following definitions.

Definition 2.1. The Lane-Emden fractional system (1.1) has the Ulam-Hyers stability if there exists a positive constant $K$ with the following property:

For every $\epsilon>0$, for any $t \in J$, and $\left(x_{1}, x_{2}, \ldots, x_{n}\right) \in S$ of (1.1), with

$$
\left|D^{\beta_{k}}\left(D^{\alpha_{k}}+\frac{a_{k}}{t}\right) x_{k}(t)+f_{k}\left(t, x_{1}(t), x_{2}(t), \ldots, x_{n}(t)\right)-g_{k}(t)\right|<\epsilon,
$$

and $k=1, \ldots, n$, then, there exists a solution $\left(y_{1}, y_{2}, \ldots, y_{n}\right) \in S$ that satisfies

$$
D^{\beta_{k}}\left(D^{\alpha_{k}}+\frac{a_{k}}{t}\right) y_{k}(t)+f_{k}\left(t, y_{1}(t), y_{2}(t), \ldots, y_{n}(t)\right)=g_{k}(t), \quad k=1, \ldots, n
$$

and

$$
\begin{aligned}
\sum_{k=1}^{n}\left|y_{k}(0)\right| & =\sum_{k=1}^{n}\left|y_{k}^{\prime}(0)\right|=\cdots=\sum_{k=1}^{n}\left|y_{k}^{(l-1)}(0)\right| \\
& =\sum_{k=1}^{n}\left|D^{\alpha_{k}} y_{k}(0)\right|=\sum_{k=1}^{n}\left|D^{\alpha_{k+1}} y_{k}(0)\right|=\cdots=\sum_{k=1}^{n}\left|D^{\alpha_{k+l-2}} y_{k}(0)\right|=0
\end{aligned}
$$

where $D^{\alpha_{k+l-1}} y_{k}(1)=0$, such that,

$$
\left\|\left(x_{1}-y_{1}, x_{2}-y_{2}, \ldots, x_{n}-y_{n}\right)\right\|_{S}<K \epsilon .
$$

Definition 2.2. The system (1.1) is Ulam-Hyers stable in the generalized sense if there exists $\varphi \in C\left(\mathbb{R}^{+}, \mathbb{R}^{+}\right)$, such that $\varphi(0)=0$ and for each $\epsilon>0$, and for each solution $\left(x_{1}, x_{2}, \ldots, x_{n}\right) \in S$ of $(1.1)$, with $\mid D^{\beta_{k}}\left(D^{\alpha_{k}}+\frac{\alpha_{k}}{t}\right) y_{k}(t)+f_{k}\left(t, x_{1}(t), x_{2}(t), \ldots, x_{n}(t)\right)-$ $g_{k}(t) \mid<\epsilon$, and $k=1, \ldots, n$, there exists $\left(y_{1}, y_{2}, \ldots, y_{n}\right) \in S$ of $(2.8)$, with

$$
\left\|\left(x_{1}-y_{1}, x_{2}-y_{2}, \ldots, x_{n}-y_{n}\right)\right\|_{S}<\varphi(\epsilon) .
$$

Definition 2.3. The system (1.1) is stable in the sense of Ulam-Hyers-Rassias if there exist $\Psi \in C\left(J, \mathbb{R}^{+}\right)$and some positive $\rho_{i}$, such that for each $\epsilon_{i}>0, i=1, \ldots n$, and for all solution $\left(x_{1}, x_{2}, \ldots, x_{n}\right) \in S$ of the inequalities

$$
\left\{\begin{array}{c}
\left|D^{\beta_{1}}\left(D^{\alpha 1}+\frac{a_{1}}{t}\right) x_{1}(t)+f_{1}\left(t, x_{1}(t), x_{2}(t), \ldots, x_{n}(t)\right)-g_{1}(t)\right| \leq \epsilon_{1} \Psi(t) \\
\left|D^{\beta_{2}}\left(D^{\alpha_{2}}+\frac{a_{2}}{t}\right) x_{2}(t)+f_{2}\left(t, x_{1}(t), x_{2}(t), \ldots, x_{n}(t)\right)-g_{2}(t)\right| \leq \epsilon_{2} \Psi(t) \\
\vdots \\
\left|D^{\beta_{n}}\left(D^{\alpha_{n}}+\frac{a_{n}}{t}\right) x_{n}(t)+f_{n}\left(t, x_{1}(t), x_{2}(t), \ldots, x_{n}(t)\right)-g_{n}(t)\right| \leq \epsilon_{n} \Psi(t)
\end{array}\right.
$$

there exists $\left(y_{1}, y_{2}, \ldots, y_{n}\right) \in S$ of $(2.8)$ that satisfies

$$
\left\|\left(x_{1}-y_{1}, x_{2}-y_{2}, \ldots, x_{n}-y_{n}\right)\right\|_{S} \leq \rho \epsilon \Psi(t), \quad \rho:=\max _{1 \leq k \leq n} \rho_{k}, \quad \epsilon:=\max _{1 \leq k \leq n} \epsilon_{k} .
$$


Remark 2.1. An element $\left(x_{1}, x_{2}, \ldots, x_{n}\right)$ of $S$ is a solution of (1.1) if and only if there exists $h_{k}:[0,1] \rightarrow \mathbb{R}, k=1,2, \ldots, n$, such that

(i) $\left|h_{k}(t)\right|<\epsilon_{k}, t \in[0,1],\left(\epsilon_{k}>0\right.$, and is sufficient small $)$, and

(ii) $D^{\beta_{k}}\left(D^{\alpha_{k}}+\frac{a_{k}}{t}\right) x_{k}(t)+f_{k}\left(t, x_{1}(t), x_{2}(t), \ldots, x_{n}(t)\right)=g_{k}(t)+h_{k}(t), t \in[0,1]$.

Theorem 2.3. Under the assumptions of Theorem 2.1, if the inequalities

$$
\sup _{t \in J}\left|D^{\beta_{k}}\left(D^{\alpha_{k}}+\frac{a_{k}}{t}\right) x_{k}(t)\right| \geq\left(M_{k}+L_{k}\right) \digamma_{k}+\left(\Sigma_{k} \digamma_{k}+a_{k} \Lambda_{k}\right) r, \quad \Sigma_{k}<1,
$$

are satisfied, then the Lane-Emden problem (1.1) is Ulam-Hyers stable in S.

Proof. Thanks to Theorem 2.1, we can state that the problem (1.1) has a solution $\left(y_{1}, y_{2}, \ldots, y_{n}\right) \in S$ that satisfies $(2.8)$.

Now, suppose $\left(x_{1}, x_{2}, \ldots, x_{n}\right) \in S$ is a solution of (1.1), where

$$
\left|D^{\beta_{k}}\left(D^{\alpha_{k}}+\frac{a_{k}}{t}\right) x_{k}(t)+f_{k}\left(t, x_{1}(t), x_{2}(t), \ldots, x_{n}(t)\right)-g_{k}(t)\right|<\epsilon_{k} .
$$

According to assumptions of Theorem 2.1, we have

$$
\left|x_{k}(t)\right| \leq\left(M_{k}+L_{k}\right) \digamma_{k}+\left(\Sigma_{k} \digamma_{k}+a_{k} \Lambda_{k}\right) r, \quad k=1,2, \ldots, n,
$$

where $\epsilon_{k}>0$.

Then, by (2.10) and (2.12), we obtain

$$
\sup _{t \in J}\left|x_{k}(t)\right| \leq \sup _{t \in J}\left|D^{\beta_{k}}\left(D^{\alpha_{k}}+\frac{a_{k}}{t}\right) x_{k}(t)\right| .
$$

Using (2.13), we get

$$
\begin{aligned}
& \sup _{t \in J}\left|x_{k}(t)-y_{k}(t)\right| \\
& \leq \sup _{t \in J}\left|D^{\beta_{k}}\left(D^{\alpha_{k}}+\frac{a_{k}}{t}\right)\left(x_{k}(t)-y_{k}(t)\right)\right| \\
& \leq \sup _{t \in J} \mid D^{\beta_{k}}\left(D^{\alpha_{k}}+\frac{a_{k}}{t}\right) x_{k}(t)-g_{k}(t)+f_{k}\left(t, x_{1}(t), x_{2}(t), \ldots, x_{n}(t)\right) \\
& \quad-\left(D^{\beta_{k}}\left(D^{\alpha_{k}}+\frac{a_{k}}{t}\right) y_{k}(t)-g_{k}(t)+f_{k}\left(t, y_{1}(t), y_{2}(t), \ldots, y_{n}(t)\right)\right) \\
& \quad-f_{k}\left(t, x_{1}(t), x_{2}(t), \ldots, x_{n}(t)\right)+f_{k}\left(t, y_{1}(t), y_{2}(t), \ldots, y_{n}(t)\right) \mid
\end{aligned}
$$

Using (2.8) and (2.11), we obtain 


$$
\begin{aligned}
\sup _{t \in J}\left|x_{k}(t)-y_{k}(t)\right| & \leq \epsilon_{k}+\left(\mu_{k}\right)_{1}\left|x_{1}(t)-y_{1}(t)\right|+\cdots+\left(\mu_{k}\right)_{n}\left|x_{n}(t)-y_{n}(t)\right| \\
& \leq \epsilon_{k}+\sum_{j=1}^{n}\left(\mu_{k}\right)_{j} \max _{1 \leq j \leq n}\left|x_{j}(t)-y_{j}(t)\right| \\
& \leq \epsilon_{k}+\sum_{j=1}^{n}\left(\mu_{k}\right)_{j} \max _{1 \leq k \leq n}\left|x_{k}(t)-y_{k}(t)\right| .
\end{aligned}
$$

Hence,

Therefore,

$$
\max _{1 \leq k \leq n}\left\|x_{k}-y_{k}\right\|_{\infty} \leq \max _{1 \leq k \leq n} \omega_{k} \epsilon_{k}, \quad \omega_{k}:=\frac{1}{1-\Sigma_{k}}
$$

$$
\left\|\left(x_{1}-y_{1}, x_{2}-y_{2}, \ldots, x_{n}-y_{n}\right)\right\|_{S} \leq \max _{1 \leq k \leq n} \omega_{k} \epsilon_{k}:=K \epsilon, \epsilon=\max _{1 \leq k \leq n} \epsilon_{k} .
$$

Since $\Sigma_{k}<1$, we get $\omega_{k}>0$, that is $K$ is a positive constant.

From (1.4), we see that

$$
\begin{aligned}
\sum_{k=1}^{n}\left|y_{k}(0)\right| & =\sum_{k=1}^{n}\left|y_{k}^{\prime}(0)\right|=\cdots=\sum_{k=1}^{n}\left|y_{k}^{(l-1)}(0)\right|=\sum_{k=1}^{n}\left|D^{\alpha_{k}} y_{k}(0)\right| \\
& =\sum_{k=1}^{n}\left|D^{\alpha_{k+1}} y_{k}(0)\right|=\cdots=\sum_{k=1}^{n}\left|D^{\alpha_{k+l-2}} y_{k}(0)\right|=D^{\alpha_{k+l-1}} y_{k}(1)=0 .
\end{aligned}
$$

Then,

$$
\left\|\left(x_{1}-y_{1}, x_{2}-y_{2}, \ldots, x_{n}-y_{n}\right)\right\|_{S} \leq K \epsilon .
$$

Therefore, the Lane-Emden fractional system (1.1) is Ulam-Hyers stable.

Remark 2.2. Taking $\varphi(\epsilon)=K \epsilon$, we conclude that the problem (1.1) is generalized Ulam-Hyers stable.

Theorem 2.4. Assume that:

(i) The assumptions and the condition (2.2) of Theorem 2.1 are satisfied.

(ii) There exists a function $\Psi \in C\left([0,1], \mathbb{R}_{+}\right)$that satisfies $(2.9)$.

Then, the fractional system (1.1) is Ulam-Hyers-Rassias stable in $S$.

Proof. Let $\left(x_{1}, x_{2}, \ldots, x_{n}\right) \in S$ be a solution of (1.1). In virtue of Remark 2.1 and by some easy calculations, we get:

$$
\max _{1 \leq k \leq n}\left\|x_{k}-y_{k}\right\| \leq \max _{1 \leq k \leq n} \frac{\epsilon_{k} \Psi(t)}{1-\lambda_{k}}:=\max _{1 \leq k \leq n} \rho_{k} \epsilon_{k} \Psi(t) .
$$

Thus,

$$
\left\|\left(x_{1}-y_{1}, x_{2}-y_{2}, \ldots, x_{n}-y_{n}\right)\right\|_{S}=\max _{1 \leq k \leq n}\left\|x_{k}-y_{k}\right\| \leq \rho \epsilon \Psi(t)
$$

where,

$$
\rho=\max _{1 \leq k \leq n} \rho_{k}, \quad \epsilon=\max _{1 \leq k \leq n} \epsilon_{k} .
$$


The Condition (2.2) implies that $\rho_{k}=1 /\left(1-\lambda_{k}\right)>0, k=1,2, \ldots, n$. Hence, (1.1) is Ulam-Hyers-Rassias stable.

\section{Applications}

In this section, we present some examples to illustrate some applications of the main results.

Example 3.1. Consider the following system:

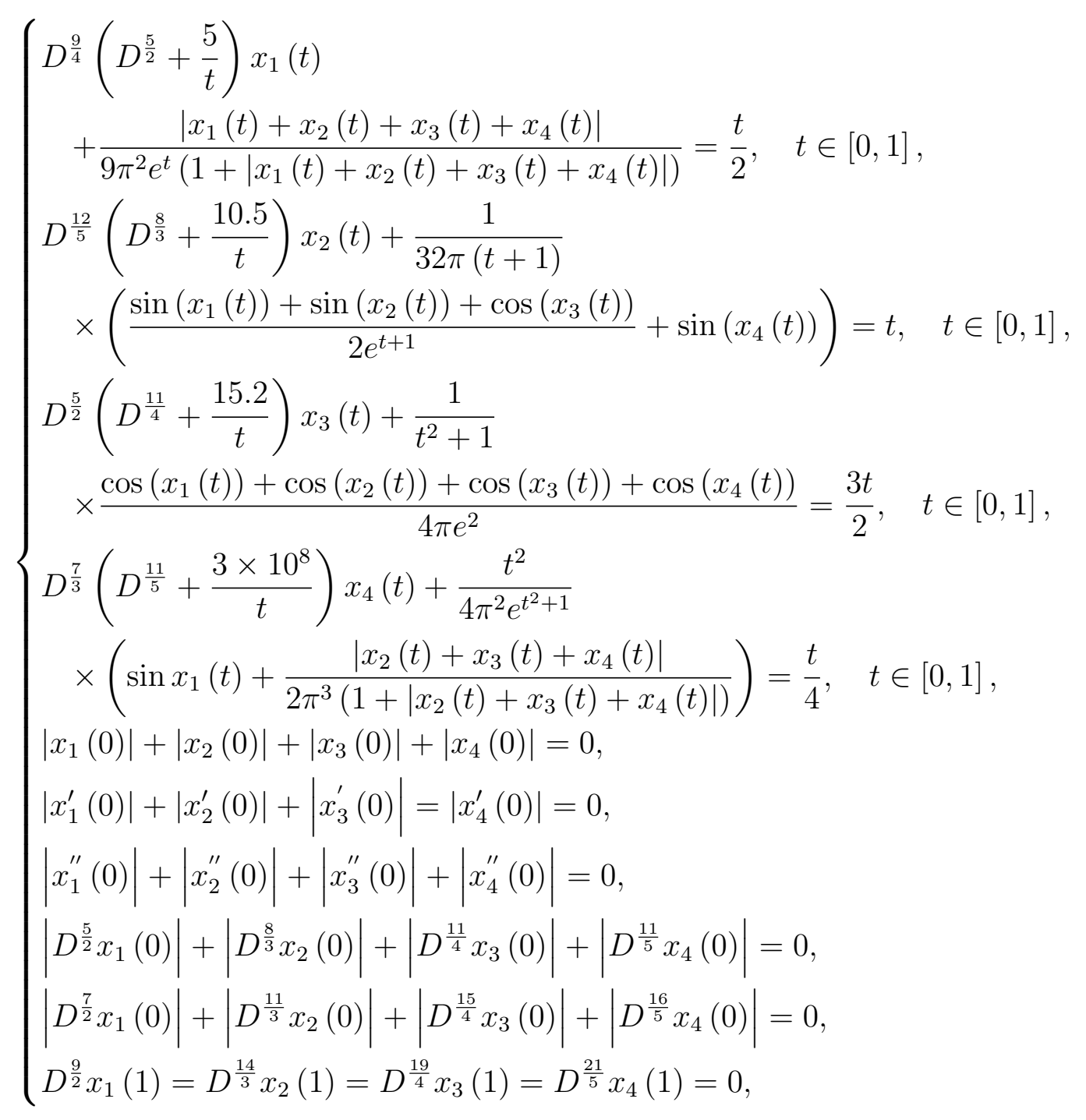

In this example, we have: $n=4, l=3, \beta_{1}=9 / 4, \beta_{2}=12 / 5, \beta_{3}=5 / 2, \beta_{4}=7 / 3$, $\alpha_{1}=5 / 2, \alpha_{2}=8 / 3, \alpha_{3}=11 / 4, \alpha_{4}=11 / 5, a_{1}=5, a_{2}=10.5, a_{3}=15.2, a_{4}=3 \times 10^{8}$, $J=[0,1]$.

It is clear that, for all $t \in[0,1]$ and $\left(x_{1}, x_{2}, x_{3}, x_{4}\right),\left(y_{1}, y_{2}, y_{3}, y_{4}\right) \in \mathbb{R}^{4}$, we have: 


$$
\begin{aligned}
& \left|f_{1}\left(t, x_{1}, x_{2}, x_{3}, x_{4}\right)-f_{1}\left(t, y_{1}, y_{2}, y_{3}, y_{4}\right)\right| \\
\leq & \frac{1}{9 \pi^{2}}\left|x_{1}-y_{1}\right|+\frac{1}{9 \pi^{2}}\left|x_{2}-y_{2}\right|+\frac{1}{9 \pi^{2}}\left|x_{3}-y_{3}\right|+\frac{1}{9 \pi^{2}}\left|x_{4}-y_{4}\right|, \\
& \left|f_{2}\left(t, x_{1}, x_{2}, x_{3}, x_{4}\right)-f_{2}\left(t, y_{1}, y_{2}, y_{3}, y_{4}\right)\right| \\
\leq & \frac{1}{64 \pi e}\left|x_{1}-y_{1}\right|+\frac{1}{64 \pi e}\left|x_{2}-y_{2}\right|+\frac{1}{64 \pi e}\left|x_{3}-y_{3}\right|+\frac{1}{32 \pi}\left|x_{4}-y_{4}\right|, \\
& \left|f_{3}\left(t, x_{1}, x_{2}, x_{3}, x_{4}\right)-f_{3}\left(t, y_{1}, y_{2}, y_{3}, y_{4}\right)\right| \\
\leq & \frac{1}{4 \pi e^{2}}\left|x_{1}-y_{1}\right|+\frac{1}{4 \pi e^{2}}\left|x_{2}-y_{2}\right|+\frac{1}{4 \pi e^{2}}\left|x_{3}-y_{3}\right|+\frac{1}{4 \pi e^{2}}\left|x_{4}-y_{4}\right|, \\
& \left|f_{4}\left(t, x_{1}, x_{2}, x_{3}, x_{4}\right)-f_{4}\left(t, y_{1}, y_{2}, y_{3}, y_{4}\right)\right| \\
\leq & \frac{1}{4 \pi^{2} e}\left|x_{1}-y_{1}\right|+\frac{1}{8 \pi^{5} e}\left|x_{2}-y_{2}\right|+\frac{1}{8 \pi^{5} e}\left|x_{3}-y_{3}\right|+\frac{1}{8 \pi^{5} e}\left|x_{4}-y_{4}\right|,
\end{aligned}
$$

and

$$
\begin{aligned}
& \sup _{t \in J}\left|g_{1}(t)\right|=\frac{1}{2}, \quad \sup _{t \in J}\left|g_{2}(t)\right|=1, \quad \sup _{t \in J}\left|g_{3}(t)\right|=\frac{3}{2}, \quad \sup _{t \in J}\left|g_{4}(t)\right|=\frac{1}{4}, \\
& \sup _{t \in J}\left|f_{1}\left(t, x_{1}, x_{2}, x_{3}, x_{4}\right)\right|=\frac{1}{9 \pi^{2}}, \quad \sup _{t \in J}\left|f_{2}\left(t, x_{1}, x_{2}, x_{3}, x_{4}\right)\right|=\frac{2 e+1}{64 \pi e}, \\
& \sup _{t \in J}\left|f_{3}\left(t, x_{1}, x_{2}, x_{3}, x_{4}\right)\right|=\frac{1}{4 \pi e^{2}}, \quad \sup _{t \in J}\left|f_{4}\left(t, x_{1}, x_{2}, x_{3}, x_{4}\right)\right|=\frac{2 \pi^{3}+1}{8 \pi^{5} e} .
\end{aligned}
$$

We can take

$$
\begin{aligned}
& \left(\mu_{1}\right)_{1}=\left(\mu_{1}\right)_{2}=\left(\mu_{1}\right)_{3}=\left(\mu_{1}\right)_{4}=\frac{1}{9 \pi^{2}}, \\
& \left(\mu_{2}\right)_{1}=\left(\mu_{2}\right)_{2}=\left(\mu_{2}\right)_{3}=\frac{1}{64 \pi e}, \\
& \left(\mu_{2}\right)_{4}=\frac{1}{32 \pi}, \\
& \left(\mu_{3}\right)_{1}=\left(\mu_{3}\right)_{2}=\left(\mu_{3}\right)_{3}=\left(\mu_{3}\right)_{4}=\frac{1}{4 \pi e^{2}}, \\
& \left(\mu_{4}\right)_{1}=\frac{1}{4 \pi^{2} e}=2\left(\mu_{4}\right)_{i},
\end{aligned}
$$

where $i=2,3,4$. In addition

$$
\begin{aligned}
& \Sigma_{1}=0.045032, \quad \Sigma_{2}=0.015436, \quad \Sigma_{3}=0.043079, \quad \Sigma_{4}=0.009769, \\
& \digamma_{1}=0.012936, \quad \digamma_{2}=0.007538, \quad \digamma_{3}=0.005478,
\end{aligned}
$$

Furthermore, we have:

$$
\lambda_{1}=0.082888<1, \quad \lambda_{2}=0.115879<1, \quad \lambda_{3}=0.136702<1, \quad \lambda_{4}=0.000793<1 .
$$

Then, by Theorem 2.1, the fractional coupled system (3.1) has a unique solution on $[0,1]$. 
To illustrate the second main result, we consider the following example:

Example 3.2.

$$
\left\{\begin{array}{l}
D^{\frac{10}{3}}\left(D^{\frac{7}{2}}+\frac{10^{2}}{t}\right) x_{1}(t)+\frac{\left|x_{1}(t)+x_{2}(t)\right|}{e^{6(t+1)}\left(1+\left|x_{1}(t)+x_{2}(t)\right|\right)}=\frac{e^{t}}{2}+t, \quad t \in[0,1] \\
D^{\frac{22}{7}}\left(D^{\frac{15}{4}}+\frac{5 \times 10^{3}}{t}\right) x_{2}(t) \\
\quad+\frac{1}{12 \pi^{2}\left(t^{2}+1\right)}\left(\cos \left(x_{1}(t)\right)+\cos \left(x_{2}(t)\right)\right)=t^{2}, \quad t \in[0,1] \\
\left|x_{1}(0)\right|+\left|x_{2}(0)\right|=\left|x_{1}^{\prime}(0)\right|+\left|x_{2}^{\prime}(0)\right|=0 \\
\left|x_{1}^{\prime \prime}(0)\right|+\left|x_{2}^{\prime \prime}(0)\right|=\left|x_{1}^{\prime \prime \prime}(0)\right|+\left|x_{2}^{\prime \prime \prime}(0)\right|=0, \\
\left|D^{\frac{7}{2}} x_{1}(0)\right|+\left|D^{\frac{15}{4}} x_{2}(0)\right|=\left|D^{\frac{9}{2}} x_{1}(0)\right|+\left|D^{\frac{19}{4}} x_{2}(0)\right|=0 \\
\left|D^{\frac{11}{2}} x_{1}(0)\right|+\left|D^{\frac{23}{4}} x_{2}(0)\right|=0 \\
D^{\frac{13}{2}} x_{1}(1)=D^{\frac{27}{4}} x_{2}(1)=0 .
\end{array}\right.
$$

For this example, we have: $n=2, l=4, \beta_{1}=10 / 3, \beta_{2}=22 / 7, \alpha_{1}=7 / 2, \alpha_{2}=15 / 4$, $a_{1}=10^{2}, a_{2}=5 \times 10^{3}, J=[0,1]$.

We see that, for all $t \in[0,1]$ and $\left(x_{1}, x_{2}\right),\left(y_{1}, y_{2}\right) \in \mathbb{R}^{2}$, we get:

$$
\begin{aligned}
& \left|f_{1}\left(t, x_{1}, x_{2}\right)-f_{1}\left(t, y_{1}, y_{2}\right)\right| \leq \frac{1}{e^{6}}\left|x_{1}-y_{1}\right|+\frac{1}{e^{6}}\left|x_{2}-y_{2}\right|, \\
& \left|f_{2}\left(t, x_{1}, x_{2}\right)-f_{2}\left(t, y_{1}, y_{2}\right)\right| \leq \frac{1}{12 \pi^{2}}\left|x_{1}-y_{1}\right|+\frac{1}{12 \pi^{2}}\left|x_{2}-y_{2}\right|,
\end{aligned}
$$

and

$$
\begin{array}{ll}
\sup _{t \in J}\left|g_{1}(t)\right|=\frac{e+2}{2}, & \sup _{t \in J}\left|g_{2}(t)\right|=1, \\
\sup _{t \in J}\left|f_{1}(t)\right|=\frac{1}{e^{6}}, & \sup _{t \in J}\left|f_{2}(t)\right|=\frac{1}{12 \pi^{2}} .
\end{array}
$$

We can take:

In addition:

$$
\left(\mu_{1}\right)_{1}=\left(\mu_{1}\right)_{2}=\frac{1}{e^{6}}, \quad\left(\mu_{2}\right)_{1}=\left(\mu_{2}\right)_{2}=\frac{1}{12 \pi^{2}} .
$$

$$
\Sigma_{1}=0.004958, \quad \Sigma_{2}=0.016887
$$

Moreover, we obtain:

$$
C_{1}=0.000042<1, \quad C_{2}=0.000654<1 .
$$

The functions $f_{k}:[0,1] \times \mathbb{R}^{2} \rightarrow \mathbb{R}$ and $g_{k}:[0,1] \rightarrow \mathbb{R}$ are also continuous. So, by Theorem 2.2, the system (3.2) has at least one solution on $[0,1]$. 


\section{REFERENCES}

[1] Z. Dahmani and S. Belarbi, Solvability for a nonlinear coupled system of $n$ fractional differential equations, Matematika (Johor Bahru) 30 (2014), 123-133.

[2] Z. Dahmani and L. Tabharit, Solvability for a BVP with Caputo derivative, J. Interdiscip. Math. (accepted).

[3] Z. Dahmani and L. Tabharit, Fractional order differential equations involving Caputo derivative, Theory Appl. Math. Comput. Sci. 4 (2014), 40-55.

[4] Z. Dahmani and A. Taieb, New existence and uniqueness results for high dimensional fractional differential systems, Facta Univ. Ser. Math. Inform. 30 (2015), 281-293.

[5] Z. Dahmani and A. Taieb, Solvability of a coupled system of fractional differential equations with periodic and antiperiodic boundary conditions, Pure and Applied Mathematics Letters 15 (2015), 29-36.

[6] Q. Dai and C. Tisdell, Non-degeneracy of positive solutions to homogeneous second order differential systems and its applications, Acta Math. Sci. 29B (2009), 437-448.

[7] R. Dalmasso, Existence and uniqueness of solutions for a semilinear elliptic system, International Journal Mathematics Mathematical Sciences 10 (2005), 1507-1523.

[8] W. Deng, Smoothness and stability of the solutions for nonlinear fractional differential equations, Nonlinear Anal. 72 (2010), 1768-1777.

[9] R. Emden, Gaskugeln, Teubner, Leipzig and Berlin, 1907.

[10] M. Gaber and M. G. Brikaa, Existence results for a coupled system of nonlinear fractional differential equation with three point boundary conditions, Journal of Fractional Calculus and Applications 3 (2012), 1-10.

[11] M. Houas and M. Benbachir, Existence and uniqueness results for a nonlinear differential equations of arbitrary order, International Journal of Nonlinear Analysis and Applications 2 (2015), 24-42.

[12] M. Houas and Z. Dahmani, New fractional results for a boundary value problem with Caputo derivative, Int. J. Open Probl. Comput. Sci. Math. 6 (2013), 30-42.

[13] M. Houas and Z. Dahmani, New results for a Caputo boundary value problem, American Journal of Computational and Applied Mathematics 3 (2013), 143-161.

[14] M. Houas and Z. Dahmani, New results for a coupled system of fractional differential equations, Facta Univ. Ser. Math. Inform. 28 (2013), 133-150.

[15] M. Houas and Z. Dahmani, Coupled systems of integro-differential equations involving RiemannLiouville integrals and Caputo derivatives, Acta Univ. Apulensis Math. Inform. 28 (2014), $133-150$.

[16] M. Houas and Z. Dahmani, New results for a system of two fractional differential equations involving $n$ Caputo derivatives, Kragujevac J. Math. 38 (2014), 283-301.

[17] M. Houas, Z. Dahmani and M. Benbachir, New results for a boundary value problem for differential equations of arbitrary order, International Journal of Modern Mathematical Sciences 7 (2013), 195-211.

[18] R. W. Ibrahim, Stability of a fractional differential equation, Miskolc Math. Notes 13 (2012), $39-52$.

[19] R. W. Ibrahim, Stability of a fractional differential equation, International Journal of Mathematical, Computational, Physical and Quantum Engineering 7 (2013), 300-305.

[20] R. W. Ibrahim, Ulam stability of boundary value problem, Kragujevac J. Math. 37 (2013), $287-297$.

[21] W. H. Jiang, Solvability for a coupled system of fractional differential equations at resonance, Nonlinear Anal. Real World Appl. 13 (2012), 2285-2292.

[22] A. A. Kilbas and S. A. Marzan, Nonlinear differential equation with the Caputo fraction derivative in the space of continuously differentiable functions, Differ. Equ. 41 (2005), 84-89. 
[23] A. Klafter, S. C. Lim and R. Metzler, Fractional Dynamics, Recent Advances, World Scientific, Singapore, 2011.

[24] M. A. Krasnoselskii, Some problems of nonlinear analysis, Amer. Math. Soc. Transl. Ser. 210 (1958), 345-409.

[25] V. Lakshmikantham and A. S. Vatsala, Basic theory of fractional defferential equations, Nonlinear Anal. 69 (2008), 2677-2682.

[26] M. Li and Y. Liu, Existence and uniqueness of positive solutions for a coupled system of nonlinear fractional differential equations, Open Journal of Applied Sciences 3 (2013), 53-61.

[27] S. M. Mechee and N. Senu, The existence of ground states to a weakly coupled elliptic system, Nonlinear Anal. 48 (2002), 905-925.

[28] S. M. Mechee and N. Senu, Numerical study of fractional differential equations of Lane-Emden type by method of collocation, Applied Mathematics 3 (2012), 851-856.

[29] S. M. Mechee, K. Tabatabaei and E. Celik, The numerical method for solving differential equations of Lane-Emden type by pade approximation, Discrete Dyn. Nat. Soc. 2011 (2011), $1-9$.

[30] S. A. Okunuga, J. O. Ehigie and S. A. Sofoluwe, Treatment of Lane-Emden type equations via second derivative backward differentiation formula using boundary value technique, in: Proceedings Of The World Congress On Engineering, July 4 - 6, London, U.K., 2012, p. 224.

[31] L. Podlubny, Fractional Differential Equations, Academic Press, New York, 1999.

[32] J. Serrin and H. Zou, Existence of positive solutions of Lane-Emden system, Atti Sem. mat. Fis. Univ. Modena 46 (1998), 369-380.

[33] A. Taieb and Z. Dahmani, A coupled system of nonlinear differential equations involing $m$ nonlinear terms, Georgian Math. J. (accepted).

[34] J. Wang, L. Lv and Y. Zhou, Ulam stability and data dependence for fractional differential equations with Caputo derivative, Electron. J. Qual. Theory Differ. Equ. 63 (2011), 1-10.

[35] A. Yildirim and T. Ozi, Solutions of singular IVPs of Lane-Emden type by the variational iteration method, Nonlinear Anal. 70 (2009), 2480-2484.

[36] H. Zou, A priori estimates for a semilinear elliptic system without variational structure and their applications, Math. Ann. 323 (2002), 713-735.

${ }^{2}$ LPAM, FACULTY SEI,

UMAB, Mostaganem, Algeria

E-mail address: taieb55550yahoo.fr

${ }^{1}$ LPAM, FACUlty SEI,

UMAB Mostaganem, Algeria

E-mail address: zzdahmani@yahoo.fr 